\title{
Diagnosing preoperative hyperglycemia in non-diabetic patients: a challenge and an opportunity
}

\author{
Helen Bui, MD • Roupen Hatzakorzian, MD • \\ Thomas Schricker, MD
}

Received: 30 January 2011/ Accepted: 24 February 2011/Published online: 4 May 2011

(C) Canadian Anesthesiologists' Society 2011

\section{To the Editor,}

We read with interest the article by Abdelmalak et al. published recently in the Journal. ${ }^{1}$ Using retrospective registry data, the authors report the prevalence of undiagnosed diabetes in a large cohort of patients undergoing non-cardiac surgery. We commend the authors for their work on such a sizeable study population but would like to comment on three issues raised in the manuscript.

First, the authors based the diagnosis of impaired fasting glucose (IFG) and diabetes mellitus (DM) on their patients' most recent plasma glucose value prior to surgery; however, it remains unclear if the study patients were fasting at the time of blood glucose measurements and, if they were, for how long. The American Diabetes Association (ADA) criterion for the diagnosis of DM using fasting plasma glucose requires a fasting period of at least eight hours. ${ }^{2}$ Hence, not knowing the duration of fasting makes it difficult to differentiate between elevated, impaired, and normal fasting glucose.

Second, the ADA stipulates that any method used to diagnose IFG or DM should be repeated at a later date to rule out laboratory error. Although this step was acknowledged by the authors, we would like to emphasize that a variable length of fasting as well as stress-induced changes in glycemia likely account for some of the abnormal plasma glucose levels found in this study.

\section{H. Bui, MD}

McGill University Health Centre, Montreal Children's Hospital,

Montreal, QC, Canada

R. Hatzakorzian, MD $(\bowtie) \cdot$ T. Schricker, MD

Royal Victoria Hospital, Montreal, QC, Canada

e-mail: roupenhatz@hotmail.com;

roupen.hatzakorzian@muhc.mcgill.ca
Measurement of hemoglobin A1c in future studies may further clarify the diagnosis.

It is well documented that a large portion of adults with type 2 diabetes are unaware of their condition. ${ }^{3}$ This article does not state the actual manner in which each patient's diabetic status was determined. It is not uncommon for patients with confirmed diabetic blood glucose levels not to consider themselves diabetic when being interviewed. It is therefore likely that a database registry underestimates the prevalence of DM, and this may account for a portion of the $10 \%$ DM reported in this study.

We performed a prospective study in almost 500 patients (fasting $>$ eight hours) before surgery and showed that $26 \%$ of previously undiagnosed patients demonstrated blood glucose levels in the IFG or DM range. ${ }^{4}$ Similar to Abdelmalak et al.'s findings, male sex and older age were associated with a higher risk of hyperglycemia. Our study population, however, had a lower mean body mass index.

We agree with the authors that pre-surgical identification of IFG and DM may be useful for predicting and influencing postoperative outcomes. We understand the inherent limitations of retrospective data analyses but caution against the premature labelling of patients as prediabetic and diabetic.

Competing interests None declared. 
3. Karve A, Hayward RA. Prevalence, diagnosis, and treatment of impaired fasting glucose and impaired glucose tolerance in nondiabetic U.S. adults. Diabetes Care 2010; 33: 2355-9.

4. Hatzakorzian R, Bui H, Carvalho G, Pi Shan WL, Sidhu S, Schricker T. Fasting blood glucose levels in patients presenting for elective surgery. Nutrition 2010; DOI:10.1016/j.nut.2010. 02.003 .

\section{Reply}

We thank Drs. Bui, Hatzakorzian, and Schricker for their interest in our manuscript, "The prevalence of undiagnosed diabetes in non-cardiac surgery patients, an observational study", 1 and for their kind comments. They raised excellent points highlighting the limitations of retrospective trials in general - including ours - many of which we have addressed in our manuscript. ${ }^{1}$

In spite of the limitations of our retrospective study, our findings were similar to those in the prospective trial involving 500 patients that was carefully conducted by Drs. Bui, Hatzakorzian, and Schricker. ${ }^{2}$ In our retrospective study involving almost 40,000 patients, the prevalence of diabetes was 14\%; this prevalence was similar to their findings.

Moreover, they found that $26 \%$ of previously undiagnosed diabetics had abnormally high blood glucose concentrations, which was comparable with our prevalence of $21 \%$. In both studies, age and sex were found to be risk factors for hyperglycemia. In addition, we found that American Society of Anesthesiologists physical status and body mass index were also risk factors, which perhaps was a result of increased precision due to a larger sample size. Therefore, the findings of these two independently conducted, methodologically divergent studies are remarkably similar.

Drs. Bui, Hatzakorzian, and Schricker's point is very well taken in cautioning against premature labelling of patients as being diabetics or pre-diabetics. However, one aim of our manuscript was to highlight the opportunity available at the preoperative evaluation process to detect and diagnose prediabetes and diabetes, as early diagnosis and treatment could result in modifying its natural course and delaying its associated complications. ${ }^{3,4}$ Increased awareness of the high proportion of hyperglycemic non-diabetic surgical patients should bolster clinicians' attention to this condition, lead to better monitoring, and direct future research in perioperative glucose control to include this particular group of patients. We have expanded our work in this population by examining approximately 75,000 patients to determine the association between such abnormally high preoperative glucose concentrations and surgical outcomes. While preoperative hyperglycemia, in all patients with and without prior diagnosis of diabetes does not increase immediate postoperative major morbidity and mortality, we found that it is directly related to long-term one-year mortality, ${ }^{5}$ and that relationship was more obvious in non-diabetics who were hyperglycemic than in their hyperglycemic diabetic counterparts. $^{6}$

Thus, there is clearly a need for more studies in this unique population (hyperglycemic non-diabetics) to define further their potential perioperative risks and to explore opportunities for improving their outcomes.

Competing interests None declared.

\section{References}

1. Abdelmalak B, Abdelmalak JB, Knittel J, et al. The prevalence of undiagnosed diabetes in non-cardiac surgical patients, an observational study. Can J Anesth 2010; 57: 1058-64.

2. Hatzakorzian R, Bui H, Carvalho G, Pi Shan WL, Sidhu S, Schricker $T$. Fasting blood glucose levels in patients presenting for elective surgery. Nutrition 2010; DOI:10.1016/j.nut.2010.02.003.

3. Anonymous. The effect of intensive treatment of diabetes on the development and progression of long-term complications in insulin-dependent diabetes mellitus. The Diabetes Control and Complications Trial Research Group. N Engl J Med 1993; 329: 977-86.

4. Expert Committee on the Diagnosis and Classification of Diabetes Mellitus. Report of the expert committee on the diagnosis and classification of diabetes mellitus. Diabetes Care 2003; 26 Suppl 1: S5-20.

5. Abdelmalak J, Dalton J, Christiansen E, Argalious M, Abdelmalak $B$. Pre-op blood glucose con. and post-op outcomes - one year mortality following non-cardiac surgery. Anesthesiology 2010; A720 (abstract)

6. Abdelmalak B, Knittel J, Abdelmalak J, Dalton J, Foss J. Preoperative blood glucose and outcomes following non-cardiac surgery, impact of diabetes. Anesthesiology 2010; A794 (abstract)

Basem Abdelmalak, MD

Robert Zimmerman, MD

Joseph Foss, MD

Cleveland Clinic, Cleveland, USA 